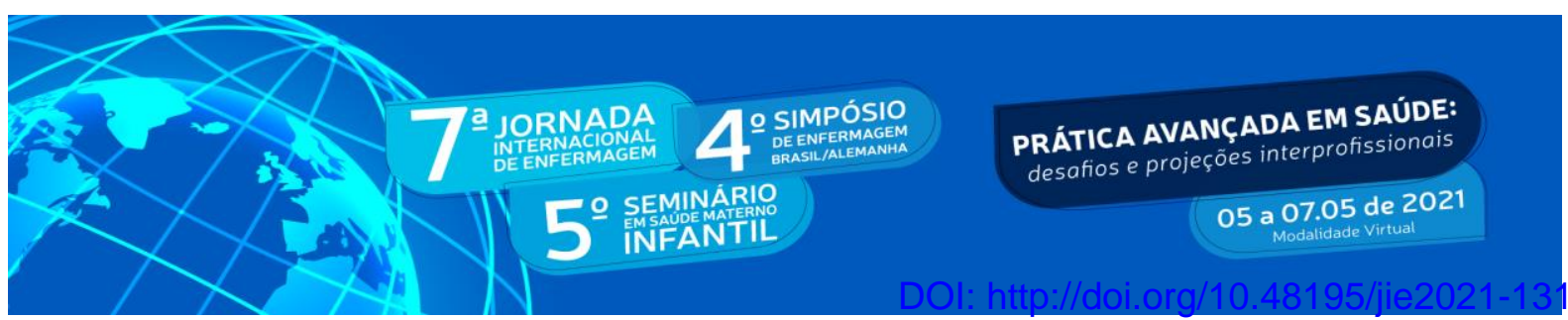

\title{
INTERVENÇÕES DE ENFERMAGEM VOLTADAS PARA A SAÚDE MENTAL DA PESSOA IDOSA ${ }^{1}$
}

\section{Eyshila Lima²; Larissa Pereira Righi da Silva ${ }^{3}$; Luiza Fortes Lamberty ${ }^{4}$; Morgana Aline da Silva ${ }^{5}$;Karine de Freitas Cáceres Machado6; Maria Helena Gehlen ${ }^{7}$.}

\begin{abstract}
RESUMO: Mediante a elaboração deste estudo objetivou-se promover e relacionar intervenções de enfermagem realizadas com idosos assistidos em uma unidade psiquiátrica de uma instituição de saúde. Trata-se de um relato de experiência, transcrito a partir das vivências das acadêmicas frente ao serviço de saúde mental e em atenção integral ao idoso, no período do segundo semestre de 2020, como atividade extensionista da disciplina de Atenção Integral à Saúde Mental do Curso de Enfermagem. Para descrição das atividades foram realizadas 2 visitas por semana envolvendo ações de acolhimento e escuta, evidenciou-se nesses idosos institucionalizados a demanda de intervenções que envolvam temas psicoafetivos. Sendo assim conclui-se que, é necessário a ação dos profissionais enfermeiros para a realização e implementação de orientações e atividades que propicie a melhora na qualidade de vida e do bem-estar, visando a benfeitoria da saúde mental à pessoa idosa.
\end{abstract}

PALAVRAS-CHAVE: Enfermagem; Envelhecimento; Saúde do Idoso; Saúde Mental.

ABSTRACT: The objective of this study was to promote and relate nursing interventions performed with the elderly assisted in a psychiatric unit of a health institution. This is an experience report, transcribed from the experiences of the students facing the mental health service and comprehensive care for the elderly, during the second semester of 2020, as an extension activity of the discipline of Comprehensive Mental Health Care of the Nursing Course. To describe the activities, two visits per week were made involving welcoming and listening actions. It was evident in these institutionalized elderly people the demand for interventions that involve psycho-affective issues. Thus, it is concluded that the action of nursing professionals is necessary for the realization and implementation of guidelines and activities that provide improvement in quality of life and well-being, aiming at the improvement of mental health of the elderly.

KEYWORDS: Nursing; Aging; Health of the Elderly; Mental Health.

\footnotetext{
${ }^{1}$ Relato desenvolvido através de vivências das acadêmicas de Enfermagem no cenário prático da Saúde Mental e na Atenção Saúde do Idoso.

${ }^{2}$ Estudante do Curso de Enfermagem da Universidade Franciscana- UFN. Santa Maria, Rio Grande do Sul, Brasil. E-mail: eyshilalima2@gmail.com

${ }^{3}$ Estudante do Curso de Enfermagem da Universidade Franciscana- UFN. Santa Maria, Rio Grande do Sul, Brasil. E-mail:Larissarighi89@gmail.com

${ }^{4}$ Estudante do Curso de Enfermagem da Universidade Franciscana- UFN. Santa Maria, Rio Grande do Sul, Brasil. E-mail: Luizaflamberty@gmail.com

${ }^{5}$ Estudante do Curso de Enfermagem da Universidade Franciscana- UFN. Santa Maria, Rio Grande do Sul, Brasil. E-mail: morgana.a.dasilva@gmail.com

${ }^{6}$ Professora Mestre Especialista em Saúde Mental do Curso de Enfermagem da Universidade

Franciscana - UFN. Santa Maria, Rio Grande do Sul, Brasil. E-mail:

Karinecaceresmachado@gmail.com

${ }^{7}$ Doutora em Gerontologia Biomédica. Especialista em Saúde do Idoso. Enfermeira Docente do

Curso de Graduação em Enfermagem. Universidade Franciscana - UFN. E-mail: mah@ufn.edu.br
} 


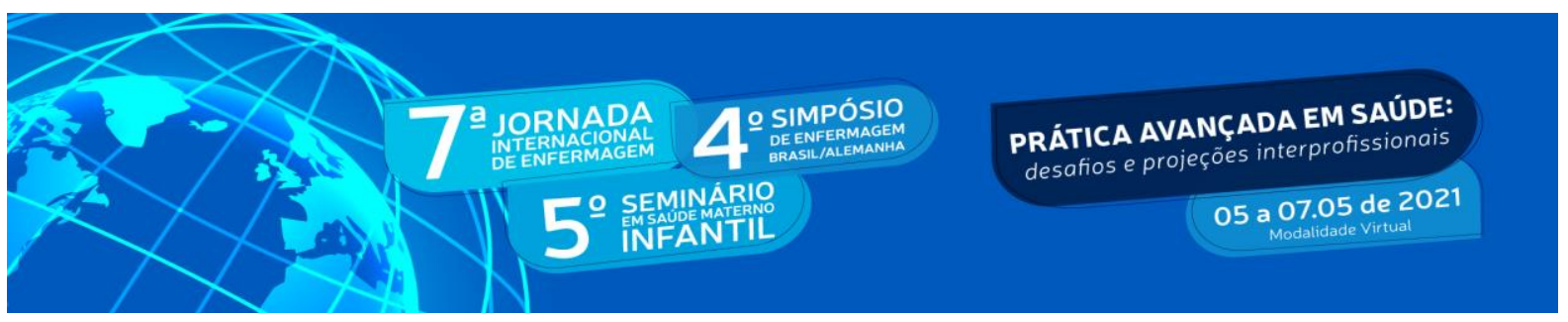

\section{INTRODUÇÃO}

O envelhecimento humano é um processo de transformação que reflete no organismo em diferentes dimensões físicas, psicológicas e no modo de pensar do próprio envelhecer. Diante dessas transformações, enfatizando as mudanças físicas, destaca-se a incidência de doenças crônicas que afetam diretamente no estado mental da pessoa idosa, levando ao aumento das perdas fisiológicas, cognitivas e sensoriais (OLIVEIRA et al., 2019).

O processo de envelhecimento populacional é uma realidade no Brasil e representa uma considerável modificação na perspectiva de vida dos indivíduos. As condições de saúde de determinada população podem ser estimadas a partir da análise das condições de vida, acompanhamento e apoio à decisão com relação às políticas públicas, investimentos em saúde e intervenções específicas. O Índice de Envelhecimento permite observar a evolução do ritmo de envelhecimento da população tornando um desafio para o futuro, garantir que os indivíduos possam envelhecer com segurança e dignidade (CLOSS; SCHWANKE, 2012).

A assistência à pessoa idosa anteriormente não era valorizada e sequer considerada como especialidade dos serviços de enfermagem. Em geral o Brasil vem enfrentando mudanças nas últimas décadas, resultado da conscientização do envelhecimento populacional e do necessário aprimoramento do serviço para fornecer uma assistência qualificada nesta faixa etária da população (DAMASCENO; SOUZA, 2018).

O país vem sofrendo com o avanço gradativo e constante na população de idosos, desta forma o impacto social deste crescimento é enorme e com isso percebemos o despreparo da população e dos profissionais de saúde frente a esta etapa da vida, uma assistência adequada às necessidades reais contribui de forma efetiva para a recuperação e manutenção da saúde, implicando na redução de complicações e na prevenção de adoecimentos mentais (MARTINHO; VARANDAS, 2017).

Segundo a Organização Mundial da Saúde - OMS (2015), o envelhecimento envolve diversas mudanças consideráveis na vida das pessoas, em especial relacionadas às alterações biológicas, senilidade, senescência, doenças e mudanças nos papéis sociais. O conflito no processo de envelhecer está em grande parte relacionado ao seu contexto social e a suas características pessoais, que repercutem no seu bem-estar físico e mental. Dentre as síndromes 


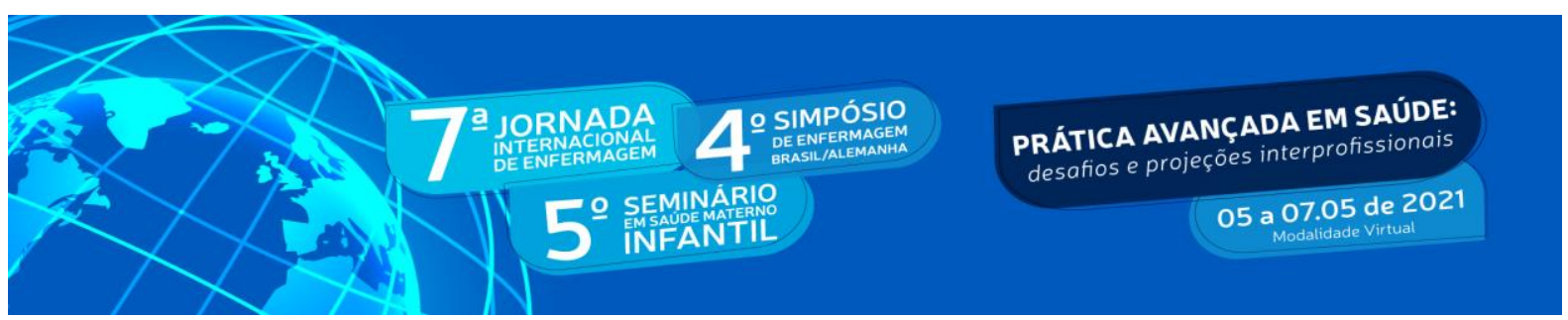

geriátricas que aparecem junto ao envelhecimento, os transtornos mentais com maior incidência entre idosos são: demência, depressão, episódios psicóticos, esquizofrenia, transtorno afetivo bipolar e transtornos ansiosos (CORREIA et al.; 2018).

Nesse contexto o cuidado à saúde mental da pessoa idosa torna-se indispensável para o envelhecimento ativo, a promoção da qualidade de vida, para fomentar a autonomia do indivíduo frente ao seu cuidado, visto que o mesmo possa manter as suas atividades básicas de vida diária e consiga viver de maneira saudável e ativa (SIVERTSEN, et al., 2015).

Em vista disso, dentre o restante dos profissionais que prestam assistência à pessoa idosa destacam-se os enfermeiros, estes possuem competências e habilidades pertencentes ao cuidado integral centrada a singularidade e as necessidades de saúde de cada indivíduo. Assim, os diagnósticos e as intervenções de enfermagem têm por fim proporcionar uma melhora na qualidade de vida, na prevenção de agravos e doenças, na promoção da saúde e da assistência através de medidas que também diminuam as limitações mentais (CORREIA et al.; 2018).

Dessa forma, mediante este estudo pretendemos promover e relacionar as intervenções de enfermagem à pessoa idosa portadora de transtornos mentais, baseados em diagnósticos de enfermagem confiáveis, com as práticas efetuadas em cenário de saúde mental e na atenção saúde do idoso no período de formação das acadêmicas do curso de Enfermagem da Universidade Franciscana, de Santa Maria - RS.

\section{OBJETIVO}

Relatar a experiência das acadêmicas de enfermagem acerca das intervenções de saúde mental realizadas para a pessoa idosa no cenário de campo prático do curso de Enfermagem.

\section{METODOLOGIA}

Trata-se de um relato de experiência, elaborado segundo vivências das acadêmicas ao exercer atividades no cenário prático de Atenção Integral à Saúde Mental e na Atenção Integral à Saúde do Idoso do curso de Enfermagem, este material foi desenvolvido a fim de contemplar as perspectivas de assistência por intermédio das intervenções de enfermagem à pessoa idosa portadora de transtornos mentais. 


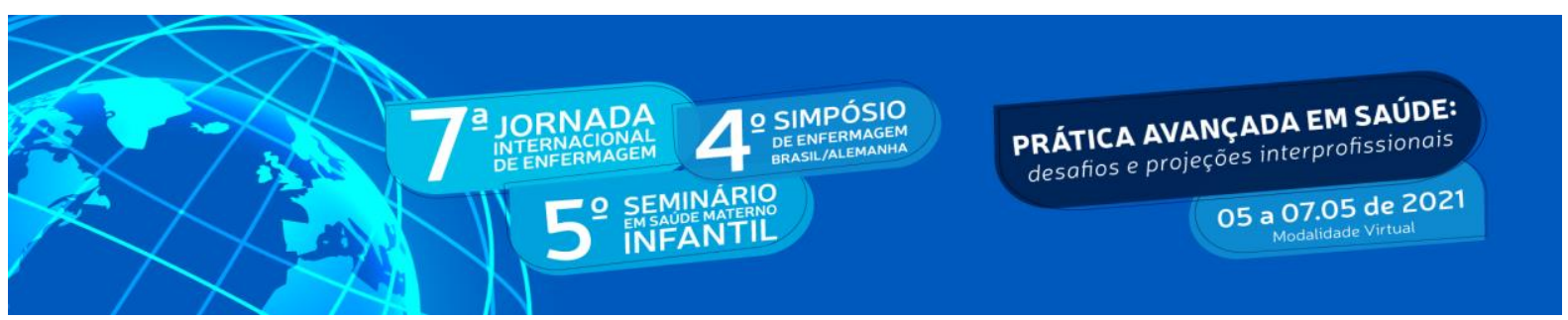

De acordo com Cortes, Padoin e Berbel (2018) esse método possibilita contribuições de ensino, visando a resolução ou minimização dos problemas evidenciados em prática, confiando ao aluno uma reflexão crítica e minuciosa dos casos.

A atividade extensionista foi realizada na unidade psiquiátrica de uma instituição de saúde, esta unidade trata pacientes de ambos os sexos sendo eles separados por setor, feminino e masculino, com área de convivência diária mista. Foi proposto para disciplina nove encontros, os quais foram realizados no período da manhã, duas vezes na semana, o que possibilitou que eles se estendessem, assim permitindo o acompanhamento do quadro dos pacientes por um maior período.

Dessa forma, este estudo foi realizado como atividade teórico-prática para a disciplina de Atenção Integral à Saúde Mental com aporte das competências adquiridas na disciplina de Atenção Integral á Saúde do Idoso do Curso de Enfermagem da Universidade Franciscana UFN de Santa Maria - Rio Grande do Sul, cursada ao longo do segundo semestre de 2020, apresentando-se como um instrumento ativo no processo de ensino aprendizagem aos estudantes do curso, com o propósito de cientificar o saber da assistência integral com a população idosa e proporcionando situações reais frente ao processo do cuidado.

\section{RESULTADOS E DISCUSSÕES}

Os atendimentos foram direcionados de acordo com as capacidades assistenciais de cada estudante no determinado período de formação, dentre as atividades ofertadas estava o acolhimento e escuta qualificada, de forma que a partir dessas atividades foi possível a percepção sobre o conhecimento dos pacientes, das suas necessidades e dos motivos pelos quais encontram-se na unidade de terapia.

O que despertou a atenção das acadêmicas para a temática foi o alto número de idosos internados na unidade psiquiátrica portadores de diversos problemas que afetam sua normalidade mental, sendo dominante a quantidade de quadros depressivos, transtornos de ansiedade e todas as características presentes nessas patologias.

É evidente a sensação de tristeza, abandono, inutilidade, dentro da sociedade e da família desses pacientes. Na percepção do assistido, a pessoa idosa pode ser vista de certa forma 


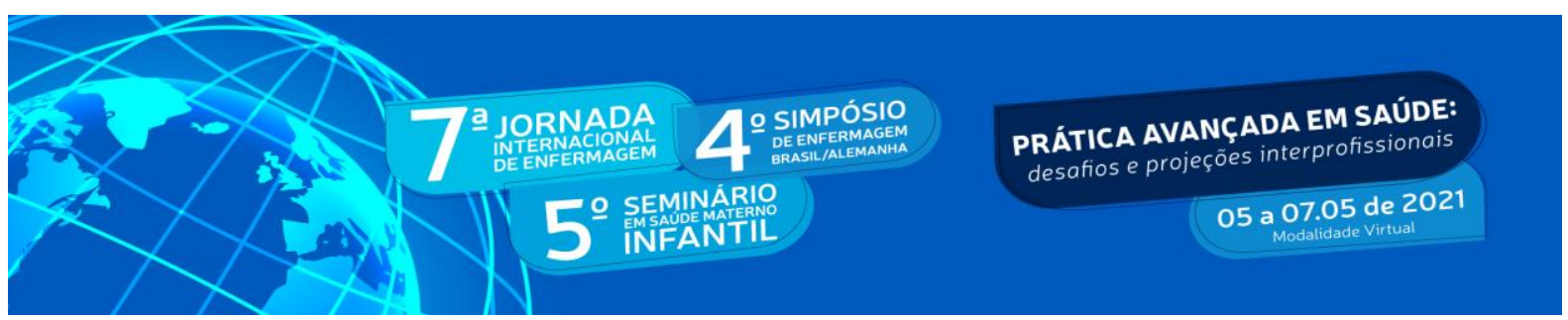

como um peso para a família, pois perdeu a capacidade de realizar as atividades cotidianas que antes eram realizadas normalmente.

Em um estudo descrito por Gullich, Duro e Cesar (2016), cujo mesmo foi realizado com 552 idosos em uma cidade rural com baixa população, percebeu-se que a prevalência de depressão nesses idosos foi de 20,4\%, sendo um número altíssimo. Percebe-se também que a maioria da população avaliada, era do sexo feminino, possuía uma renda inferior a 3 saláriosmínimos e a taxa de hospitalização a esse grupo com diagnóstico de depressão foi de 3 vezes maior do que os que não tinham esse diagnóstico. Porém, em idosos que participaram de alguma atividade de lazer, a proteção chegou a $60 \%$, sendo perceptível o auxílio das intervenções em resultado da melhora do quadro clínico.

Dessa maneira, durante as práticas foram realizadas intervenções, como uma roda de conversa com as mulheres enquanto era ofertada a oficina de manicure. Essa intervenção é de suma importância, visto que além de estabelecer vínculo e permitir que as usuárias se sintam seguras para conversar, também auxilia no crescimento da autoestima e autopercepção delas.

Percebe-se em especial que ao abordar mulheres idosas, na qual muitas vezes por conta da senescência essa percepção resulta no desprezo por sua aparência, vergonha e timidez, assim consequentemente o agravo de seus distúrbios como ao exemplo da depressão; com isso, devese instruí-las e orientá-las frente o autocuidado.

Além do mais, muitos relataram que o ato da internação se torna um fato benéfico, pois além de terem companhia, visto que a terceira idade torna-se uma fase muito solitária porque a maioria das vezes todos os familiares já partiram, a institucionalização se torna uma atividade animada através da convivência com os outros pacientes, a atenção integral da equipe multiprofissional, medicamentos na posologia correta, permitindo com que o período da internação se torne satisfatório, trazendo a sensação de cuidado e bem-estar, ademais proporcionando o sentimento de valorização como seres humanos.

Em tal caso, ao longo dos dias foi proposto os diagnósticos de enfermagem de acordo com a taxonomia NANDA (2018) e o que poderíamos fazer para intervir neste processo. De 


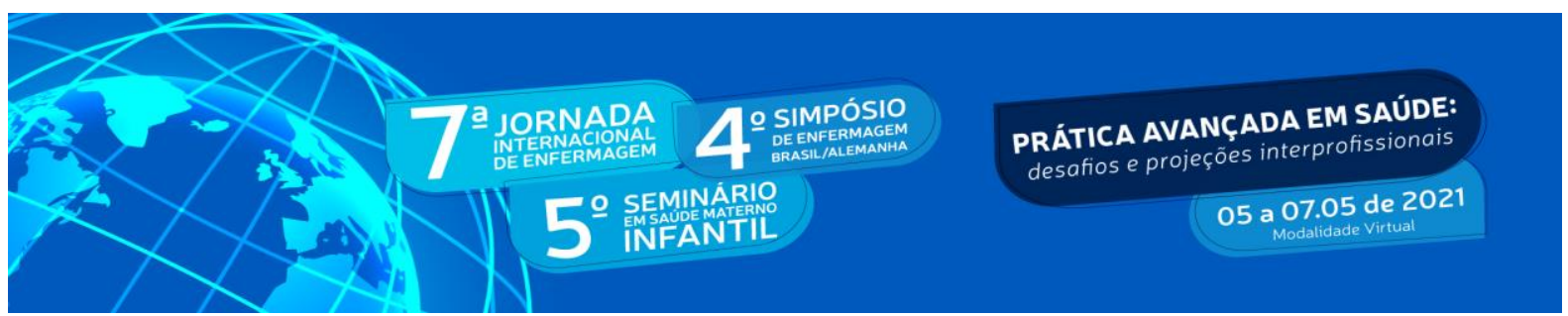

acordo com os cuidados prestados aos pacientes assistidos, um dos diagnósticos identificado no momento que ele se encontra inicialmente hospitalizado, é "processo de pensamento perturbado" e "déficit. do autocuidado; muitos pacientes vão por meio próprio, porém, os que chegam para uma internação compulsória apresentam uma grande dificuldade de se adaptar ao ambiente, ficam mais reservados em seus quartos e apresentando negatividade em seus pensamentos, pois muitas vezes chegam sem nenhum uso de medicação ou com o excesso dela.

Os idosos hospitalizados permanecem na unidade no período de 21 dias até a adaptação, por conseguinte até as medicações realizarem seus efeitos esperados. A cada dia que passa, são elaborados planejamentos terapêuticos, que visam garantir o bem-estar e melhora da qualidade de vida do usuário, momentos de reflexão e interação com os demais por meio de atividades integradas. Neste contexto é de grande valia o incentivo para a análise do seu próprio comportamento, instigando o relacionamento interpessoal e a fornecer melhores relatos da sua percepção de saúde como também os fatos que os cercam.

As intervenções realizadas são de acordo com cada diagnóstico do paciente, pensando na melhor maneira de ofertar apoio e qualidade assistencial para cada idoso. Importante salientar que grande parte dos pacientes que estão internados, não é a sua primeira institucionalização, ou seja, a grande parte já internou 2 vezes ou mais, por este motivo o vínculo que a equipe estabelece com os pacientes faz com que se sintam à vontade para expressar seus sentimentos.

Correia et al. (2018), descreve que uma grande aposta na efetividade das intervenções é resgatar e trabalhar temas psicoafetivos, frente aos transtornos mentais, pois ao utilizar estratégias de promoção da saúde mental explorando seus efeitos na redução de sintomas depressivos, a pessoa idosa pode utilizar de mecanismos como o apoio do serviço social, o suporte pessoal através de conversas e o encorajamento na realização de atividades;

Esse processo demonstrou boa aceitação dos idosos institucionalizados, gerando efeitos benéficos como aumento da esperança, confiança nas habilidades e autoconsciência para desenvolver independência e autonomia nas atividades cotidianas. 


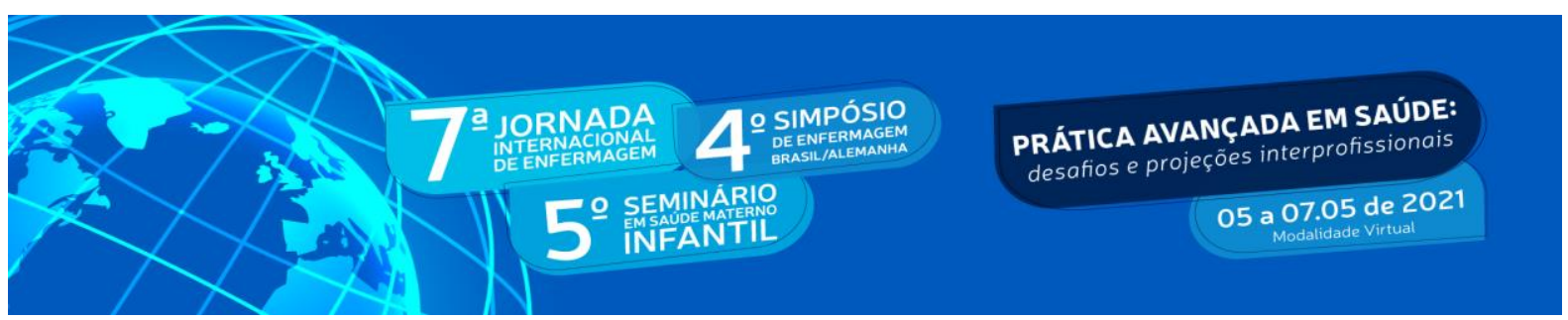

De forma geral, a saúde mental de idosos é um indicador real e significativo para a saúde do país, a enfermagem como pioneira na realização de intervenções assistenciais e de cuidado, promove ações a fim de propiciar uma qualidade de vida digna para este grupo, que se encontra perpassando pelas fases do envelhecimento, assim essa ênfase permite a utilização de terapêutica não farmacológica e demonstra a importância positiva dos cuidados à pessoa idosa com transtornos mentais (CLOSS; SCHWANKE, 2012).

\section{CONCLUSÃO}

Portanto, a partir da construção deste relato de experiência identificou-se por meio de evidências científicas a importância de intervenções para com idosos, sobretudo com os que necessitam de apoio por possuir algum quadro que afeta diretamente a sua saúde mental, visto que a prevalência de idosos com depressão e ansiedade é altíssima e está aumentando com o passar dos anos. Enquanto discentes, percebe-se a necessidade de um maior apoio a essa população, em virtude de que é deixada de lado por anos.

É notório que a enfermagem por ser uma profissão que lida diretamente com a prevenção e promoção da saúde, deverá realizar a busca por intervenções e estudos científicos que mostrem a necessidade dessa população para com práticas assistenciais e comprovadas da melhor forma, que vise a melhora da sua saúde mental, principalmente ao final de sua vida.

Contudo, esse processo deve ser visto como algo natural de todo ser humano e é importante que se viva da maneira em que o mesmo saiba a importância de se aceitar e ser feliz consigo mesmo. Por isso, faz necessário o diagnóstico precoce com essa população, para conseguir a aplicação de intervenções que venham a auxiliar nesse processo.

\section{REFERÊNCIAS}

CLOSS, V. E.; SCHWANKE, C. H. A.. A evolução do índice de envelhecimento no Brasil, nas suas regiões e unidades federativas no período de 1970 a 2010. Rev. bras. geriatr. gerontol. vol.15 no.3 Rio de Janeiro July/Sept. 2012. Disponível em: https://doi.org/10.1590/S1809-98232012000300006. Acesso em: 12/03/2021.

CORREIA, A. de S. B. et al.. Intervenções de enfermagem à pessoa idosa com transtorno mental: revisão integrativa. Anais III CONBRACIS... Campina Grande: Realize Editora, 


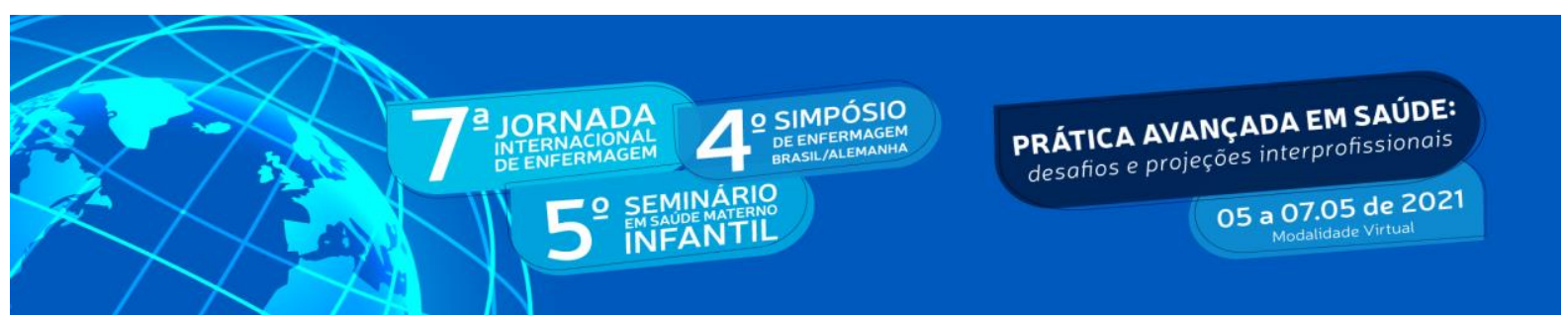

2018. Disponível em: http://www.editorarealize.com.br/artigo/visualizar/41162. Acesso em: $13 / 03 / 2021$.

CORTES, L. F.; PADOIN, S. M. de M.; BERBEL, N. A. N.. Metodologia de Problematização e Pesquisa Convergente em Saúde: proposta de práxis em pesquisa. Rev. Bras. Enferm., Brasília, v. 71, n. 2, p. 440-445, Abr. 2018 . Disponível em: http://www.scielo.br/scielo.php?script=sci_arttext\&pid=S003471672018000200440\&lng=en\&nrm=iso . Acesso em: 14/03/2021.

DAMASCENO, V.C.; SOUZA, F.S.P. Cuidado de Saúde Mental à Pessoa Idosa: Percepção do Enfermeiro. Rev Enferm UFPE on line., Recife, 12(10):2710-6, out., 2018. Disponível em: https://doi.org/10.5205/1981-8963-v12i10a234647p2710-2716-2018. Acesso em: $13 / 03 / 2021$.

GULLICH, I.; DURO, S. M. S.; CESAR, J. A. Depressão entre idosos: um estudo de base populacional no sul do Brasil. Rev Bras Epidemiol., v. 19, n 4, p. 691-701, Out-Dez 2016. Disponível em: https://doi.org/10.1590/1980-5497201600040001. Acesso em: 14/03/2021.

MARTINHO, A.F.; VARANDAS, M. de L. Impacto de um Projeto de Intervenção Comunitária nos Diagnósticos de Enfermagem. Cadernos de Saúde Vol. 9 Número único 2017, p. 35-43. Disponível em: https://doi.org/10.34632/cadernosdesaude.2017.2863 . Acesso em: $12 / 03 / 2021$.

\section{NANDA INTERNATIONAL. Diagnósticos de Enfermagem da NANDA: definições e} classificação 2018-2020. 10 ed. Artmed, 2018. Acesso em: 13/03/2021.

OLIVEIRA, L. M. et al.. Diagnósticos e intervenções de enfermagem em idosos com depressão na atenção básica. Anais VI CIEH... Campina Grande: Realize Editora, 2019. Disponível em: http://www.editorarealize.com.br/artigo/visualizar/53469. Acesso em: $12 / 03 / 2021$.

ORGANIZAÇÃO MUNDIAL DA SAÚDE. Relatório mundial sobre envelhecimento e saúde. V.1, p.1-32. 2015. Disponível em: https://www.who.int/ageing/publications/worldreport-2015/es/ Acesso em: 13/03/2021.

SILVERTSEN, H. et al. Depression and Quality of Life in Older Persons: A Review. Dement Geriatr Cogn Disord. v 40. 2015, p 311-339. Disponível em:

https://www.researchgate.net/deref/http\%3A\%2F\%2Fdx.doi.org\%2F10.1159\%2F000437299. Acesso em: 13/03/2021. 